\title{
Sistema de supervisión y automatización del proceso de clarificación de agua en la planta de tratamiento de agua potable El Dorado
}

Fernando Martínez Santa*, Javier Gamboa Torres**, Andrés Rodríguez Falla**

\section{Resumen}

El proceso de clarificación del agua que proviene del lavado de filtros en la planta El Dorado, de la Empresa de Acueducto y Alcantarillado de Bogotá (EAAB), se realiza de manera manual. Este hecho genera retardos entre los procesos y desgaste y fatiga en los equipos que acortan su vida útil. Además, no se cuenta con un sistema SCADA, lo que, de paso, muestra que el proceso de clarificación de agua se encuentra aislado. Esto ocasiona que el tiempo en planta de los operarios no sea el adecuado y afecta la calidad de los procesos posteriores. Sería, por demás, aconsejable que las condiciones de turbiedad con las que llega el agua a las corrientes naturales presentaran la menor cantidad de partículas sólidas para que no afecten de forma considerable la vida silvestre, el cauce natural y el lecho de los ríos, es decir, sin causar daños irreversibles al medio ambiente.

Palabras clave: clarificación, condiciones de turbiedad, lavado de filtros, partículas sólidas, sistema SCADA

\begin{abstract}
The process of clarifying the water that comes from the washing of filters in the El Dorado plant, of the Water and Sewerage Company of Bogota (EAAB), is done manually. This fact generates delays between processes and wear and fatigue in equipment that shorten its useful life. In addition, there is no SCADA system, which, by the way, shows that the water clarification process is isolated. This causes that the time in plant of the operators is not adequate and affects the quality of the subsequent processes. It would also be advisable for turbidity conditions under which water reaches natural currents to have the least amount of solid particles so that they do not significantly affect wildlife and the natural bed of the rivers; in other words, without causing irreversible damage to environment.
\end{abstract}

Keywords: Clarify, Filter Washing, SCADA System, Solid Particles, Turbidity Conditions

* Docente en la Facultad Tecnológica Universidad Distrital (FJC). Contacto: fmartinezs@udistrital.edu.co

** Egresado de la Facultad Tecnológica Universidad Distrital (FJC). Contacto: gamtojafer@hotmail.com

*** Egresado de la Facultad Tecnológica Universidad Distrital (FJC). Contacto: and.rof@hotmail.com 


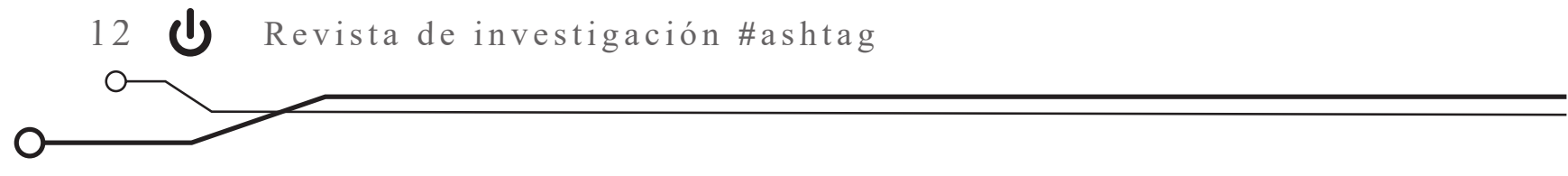

\section{Introducción}

La Empresa de Acueducto y Alcantarillado de Bogotá (ЕAAB) es una empresa pública prestadora de servicios de acueducto y alcantarillado sanitario y pluvial. Tiene 124 años de experiencia y abastece, con una de las mejores aguas del continente, cerca de 1700000 usuarios o predios en Bogotá y, además, presta servicios a 11 municipios vecinos.

En la zona sur se encuentra la planta de tratamiento El Dorado, que distribuye el agua potable a la población del suroriente de la ciudad. La planta se encuentra ubicada en la localidad de Usme. Esta se abastece del embalse La Regadera, situado a $11 \mathrm{Km}$ de la planta. Posee una capacidad de 1600 l/s y produce actualmente $400 \mathrm{l} / \mathrm{s}$ de agua potable para satisfacer la demanda de 300000 habitantes aproximadamente. La capacidad de abastecimiento de la planta es de $1,6 \mathrm{~m}^{3} / \mathrm{s}$. (Acueducto, 2015). Esta planta se ha convertido en un punto estratégico, pues el crecimiento descontrolado de la ciudad ha aumentado la demanda de los servicios que presta la empresa, especialmente en el de abastecimiento de agua potable. Por ende, la exigencia de mejoras tecnológicas en dicha planta es inminente. $\mathrm{La}$ administración de la planta ha puesto en marcha una serie de proyectos que dan cumplimiento a los requerimientos necesarios para poder satisfacer las necesidades de los usuarios nuevos.

En dicha planta hay un subproceso que se encarga de reintegrar al medio ambiente algunos de los residuos generados durante el proceso de potabilización, clarificando el agua proveniente del lavado de los filtros que se utilizan para potabilizar. Al clarificar el agua es necesario retirar la mayor cantidad de partículas sólidas y lodos inmersos en esta, pues el agua debe retornar a la quebrada en condiciones óptimas -que no afecten la quebrada y sus alrededores-.

El proceso de clarificación se realiza en tres etapas: dosificación, ecualizado y espesado. Estas se ejecutan de manera manual en la planta, hecho que exige la intervención constante de operarios que deben dividir su tiempo de trabajo y atención entre el subproceso de clarificación y los demás procesos propios de la planta en general. Bajo estas circunstancias, dicho proceso deja de ser monitoreado y controlado de manera precisa. Así, los resultados de la clarificación no son los mejores, pues, la vida útil de cada uno de los equipos se reduce considerablemente. En ocasiones, incluso se utilizan más reactivos de los necesarios y el proceso se aísla del cuarto de supervisión general de la planta.

Tras considerar esa situación, en este artículo se explica la implementación de un sistema automatizado que facilita el control y monitoreo de las variables propias del proceso de clarificación de agua en dicha planta. El empleo de esta tecnología garantiza la obtención de agua clarificada de mejor calidad, gracias a que permite un funcionamiento más eficiente de la planta. 


\section{Metodología}

\section{Caracterización de la etapa 1: canaleta Parshall}

En esta etapa, el agua ingresa a un canal que se estrecha en su parte central y vuelve a su tamaño original al final del tramo (figura 1). Este sistema es ampliamente utilizado en plantas de tratamiento para calcular el caudal de agua que ingresa a determinado proceso. Además, se suele aprovechar la turbulencia producida para dosificar algún reactivo si es necesario.

Actualmente, la canaleta Parshall, que se utiliza en esta etapa, se encuentra sin instrumentación alguna, por lo que no es posible calcular el caudal ni la turbiedad exactas con las que ingresa el agua al siguiente proceso. Además de ese problema, esta etapa previamente contaba con una bomba dosificadora de reactivo que fue retirada por deterioro. En ese sentido, también es un problema que este proceso no cuente con un sistema adecuado de dosificación para aplicar el reactivo a la corriente de agua (Reyes, 2004), pues se realiza manualmente.

Figura 1. Canaleta Parshall

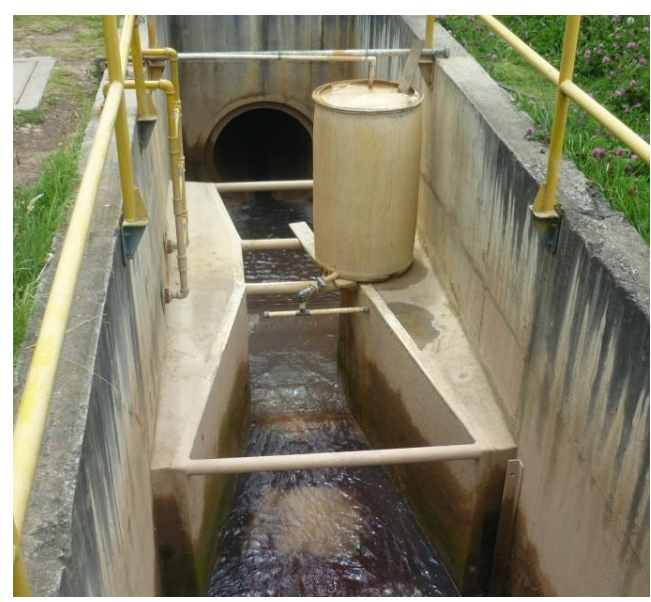

Fuente: elaboración propia

\section{Caracterización de la etapa 2: tanques ecualizadores}

El agua proveniente de la Canaleta Parshall ingresa a los dos tanques que se observan en la figura 2. En su interior, el agua sufre un proceso de aquietamiento: se deja en reposo cierto tiempo para que el reactivo agregado anteriormente y la gravedad surtan efecto. En este proceso, conocido como decantación, las partículas sólidas se unen unas con otras gracias al reactivo utilizado y, por efecto de la gravedad, se van acumulando en el fondo del tanque. Después de un tiempo y tras alcanzar la turbiedad deseada en la parte alta y parte baja del tanque -baja y alta turbiedad respectivamente-, sigue el paso del lodo, que se encuentra en el fondo, al siguiente proceso. Por su parte, el agua clara de la parte superior es enviada hacia la quebrada. Estos dos procesos se realizan con la supervisión y el criterio de un operador que debería permanecer durante el tiempo que tome el proceso; sin embargo, por la rotación de turnos, el operador puede ser relevado. 


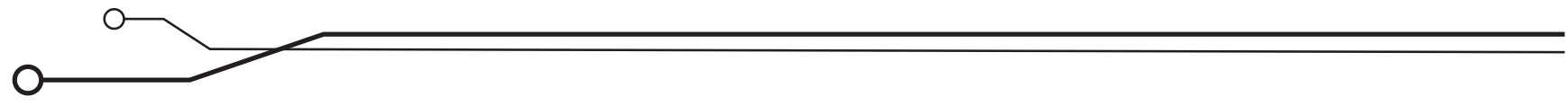

Durante la ausencia del operario suelen suceder eventos que afectan considerablemente el proceso de clarificación. Uno de ellos es que los motores encargados de bombear el lodo o el agua permanezcan encendidos sin supervisión. Esto ocasiona que, en el bombeo de lodo al final, se registre abundante agua, afectando el siguiente proceso. Una vez bombeado todo el lodo, se hace fluir el agua clarificada hacia la quebrada hasta desocupar por completo el tanque. Este hecho significa que, durante un lapso, las bombas quedan trabajando en vacío, lo que causa daños y exige mantenimientos constantes. Además de esto, los tanques no cuentan con la instrumentación necesaria para generar alarmas de niveles extremos, ni de turbiedad. Por otro lado, la instrumentación existente se encuentra en pésimas condiciones: los sensores de nivel bajo (flotadores) están deteriorados y no cumplen su función de manera adecuada.

Figura 2. Tanques para ecualizado

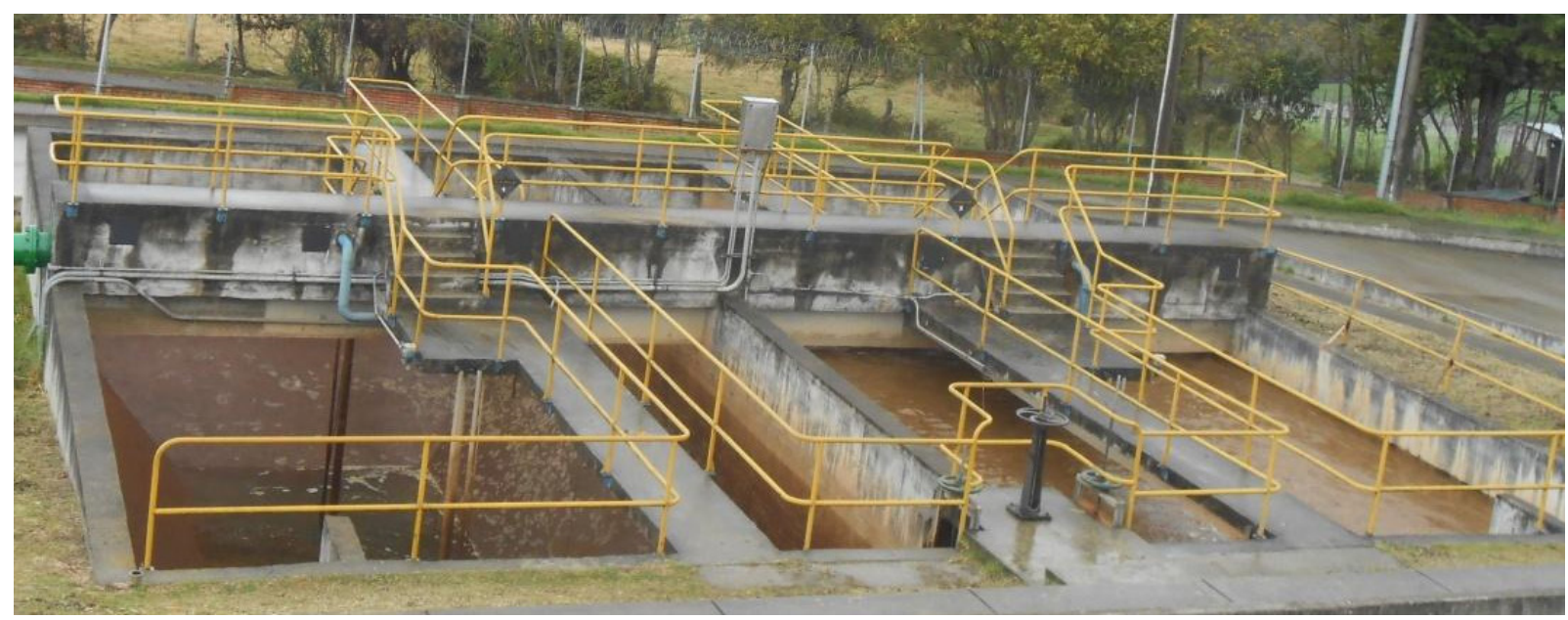

Fuente: elaboración propia

\section{Caracterización de la etapa 3: tanques espesadores}

En esta etapa, el lodo que proviene de los tanques de ecualizado se somete a un proceso de espesado. Este consiste en depositar el lodo en dos tanques de forma cónica (figura 3) para que una hélice central, que gira a velocidad constante, acumule el lodo en el fondo presionándolo para que sus partículas se condensen aún más y el agua se dirija a la parte superior del tanque. Una vez alcanzada la turbiedad requerida, el agua clarificada en la parte superior, por efecto del rebose, se dirige hacia la quebrada (Suárez et al., 2015). El lodo acumulado en el fondo debe seguir su curso hacia la segunda fase de la planta de lodos. En el proceso de espesado, el sistema de medición se hace por apertura manual de válvulas. Debido a la naturaleza del lodo se detectaron tuberías y válvulas obstruidas, además que algunas secciones están rotas. Por ende, no se tiene control sobre el agua clarificada que se espera como producto final. 
Figura 3. Tanques de espesado

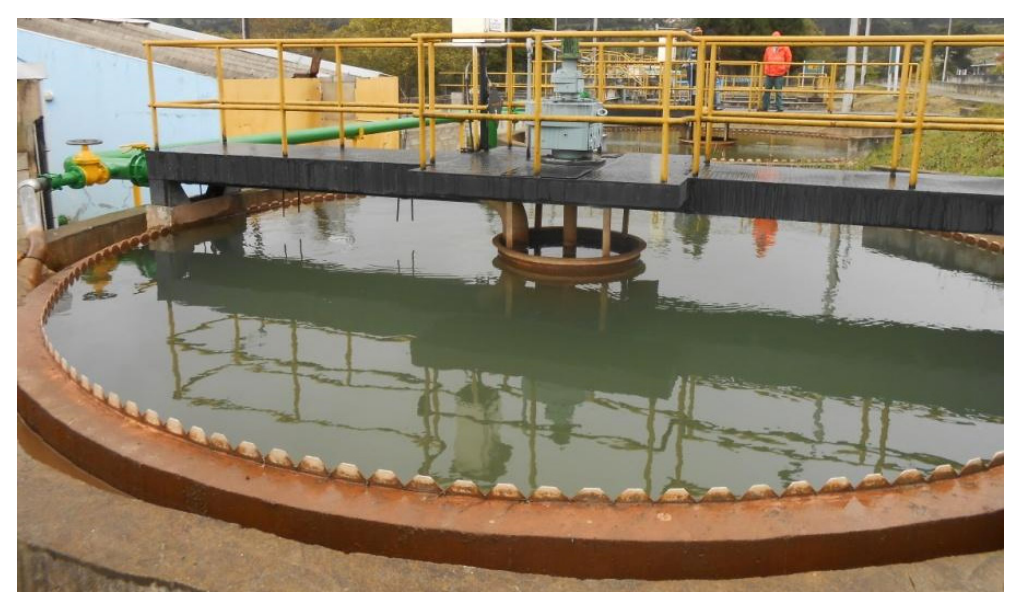

Fuente: elaboración propia

\section{Funcionamiento general de una planta de lodos}

La planta de tratamiento de lodos tiene por objeto evitar que la quebrada aledaña se contamine, pues esta recibe todos los desagües del proceso de tratamiento de agua potable, es decir, aguas provenientes del lavado de filtros, sedimentadores y floculadores. Hasta hace muy pocos años, en las estaciones de tratamiento de agua potable solo se gestionaba la producción de agua potable. En realidad, no se prestaba mucha atención a los lodos que se producían tanto en los sedimentadores como en el lavado de los filtros, pues se consideraba que, en definitiva, estos estaban formados por las sustancias que llevaban las aguas naturales, o aguas brutas, generalmente inorgánicas, como arcillas, arenas finas o limos. Hoy en día se sabe que las aguas naturales se están degradando y contaminando por diversas circunstancias. También, hay mayor conciencia y presión medioambiental y legislativa que persuaden a los gestores de los abastecimientos con la idea de construir plantas de tratamiento para estos lodos, cuyo objetivo es extraer la materia sólida y obtener unos efluentes sin lodos que puedan ser vertidos al cauce, o bien, enviados a la cabecera del tratamiento en la siguiente fase, junto al agua bruta (Ambientum., 2002).

\section{Solución implementada}

\section{Selección del controlador lógico programable (PLC)}

Se seleccionó el PLC serie Ponto PO3342 del fabricante brasileño Altus (Altus, 2011b), debido básicamente a que las instalaciones ya contaban con este controlador y con los módulos para las interfaces de comunicación Ethernet y Profibus -necesarias para la red de instrumentación de campo y el sistema de supervisión-. No fue viable cambiar el PLC debido a que el tiempo de gestión y adquisición de dichos equipos era de aproximadamente un año, lo que prolongaba el tiempo de ejecución del proyecto, además de la limitación de recursos económicos. La otra alternativa contemplada era un PLC de Telemecanique -disponible en el almacén de repuestos de 


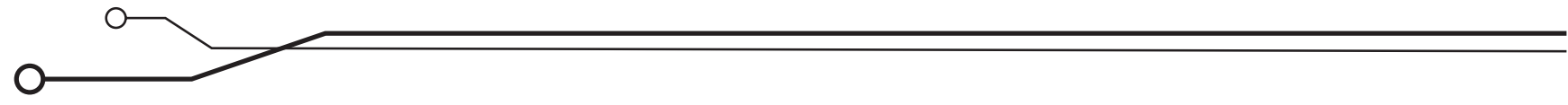

la planta-. Sin embargo, fue rechazada por su tecnología de vieja data y por no contar con el módulo de conexión Ethernet para la

\section{Selección de los sensores}

Una vez identificado el PLC a trabajar, la selección de los sensores de nivel y turbiedad se realizó teniendo en cuenta las tablas 1 y 2 . La instrumentación para sensar requería de un rango de medición mayor a 5m, 0 a 9999 NTU, comunicación PROFIBUS PA (PROcess Field BUS in Process Automation), y protección mínima al supervisión SCADA (Sistemas Digitales de Control 2002 SL, 2014).

polvo y máxima al agua, pues se debía instalar a la intemperie y muy cerca a los tanques de tratamiento. Por lo anterior, se seleccionó el sensor de nivel Prosonic M FMU40 (Endress+Hauser, 2008) y el sensor de turbiedad Liquisys M CUM 223 (Endress+Hauser, 2006).

Tabla 1. Sensores de nivel disponibles en el almacén de la planta

\begin{tabular}{|c|c|c|c|}
\hline Ref. & Rango & Com. & IP \\
\hline $\begin{array}{c}\text { VEGAPULS WL 61 } \\
\text { VEGA }\end{array}$ & $\begin{array}{c}0-15 \mathrm{~m} \\
\text { (Radar) }\end{array}$ & $4-20 \mathrm{~mA}$ & $66-68$ \\
\hline LVU 809 OMEGA & $\begin{array}{c}0-3 \mathrm{~m} \\
\text { (ultrasonido) }\end{array}$ & $4-20 \mathrm{~mA}$ & 65 \\
\hline $\begin{array}{c}\text { Prosonic M FMU40 } \\
\text { Endress+ Hauser }\end{array}$ & $\begin{array}{c}\text { Hart, ProfiBus } \\
\text { (ultrasonido) }\end{array}$ & $\begin{array}{c}\text { PA, Fieldbus } \\
\text { Foundation (San- } \\
\text { tis y Villa, 2009) }\end{array}$ & 68 \\
\hline
\end{tabular}

Fuente: elaboración propia

Tabla 2. Sensores de Turbiedad disponibles en el almacén de la planta

\begin{tabular}{|c|c|c|c|}
\hline Ref. & Rango & Com. & IP \\
\hline Liquisys M CUM 223 & $0-9999$ FNU & $\begin{array}{c}\text { Hart, Profibus PA/ } \\
\text { DP }\end{array}$ & 65 \\
\hline VISO TURB 700 YSI & $\begin{array}{c}0,05-4000 \\
\text { FNU }\end{array}$ & $\begin{array}{c}\text { Fielbus } \\
\text { Foundation }\end{array}$ & 68 \\
\hline SOLITAX sc HACH & $\begin{array}{c}0,001-4000 \\
\text { FNU }\end{array}$ & $\begin{array}{c}\text { 4-20ma, MODBUS } \\
\text { (Tecnología Digital } \\
\text { del Bajío, 2012) }\end{array}$ & 67 \\
\hline
\end{tabular}




\section{Intervención en la etapa 1}

Como se expuso anteriormente, esta etapa no cuenta con ningún tipo de instrumentación, por lo que exige la implementación de un sensor de nivel que ayude a determinar el caudal de agua que transita por la canaleta. También se debe implementar un sensor de turbiedad, cuya lectura, y con el valor del caudal obtenido, se utilizan para comparar las condiciones del agua que ingresa a las etapas de ecualizado y espesado, y la que viene después de dichos subprocesos.

La canaleta es un sistema de aforo con una estructura hidráulica que permite medir la cantidad de agua que pasa por una sección de un determinado canal. Los medidores Parshall se nombran por el ancho de su garganta, siendo sus partes: 1) la transición de entrada, 2) la sección convergente, 3) la garganta, y 4) la sección divergente.

El aforador Parshall es una reducción de la sección del canal que obliga al agua a elevarse o a remansarse para luego volver a descender hasta el nivel inicial sin el aforador. En este proceso se presenta una aceleración del flujo que permite establecer la relación matemática entre la altura de carga, o elevación, que alcanza el agua y el caudal que circula a través del dispositivo. La única medida a tomar es la altura $h$ del nivel que alcanza el agua en un punto cercano al comienzo de la sección convergente. Luego se aplica la ecuación 1 para calcular el caudal (Lux, 2010). Sus valores se indican en la figura 4.

$$
Q=K h^{n}
$$

Ecuación 1. Cálculo del caudal en la canaleta Parshall.

Las variables de este proceso son: 1) el nivel del agua, medido dentro de la cámara de aquietamiento de la canaleta Parshall; 2) el nivel de turbiedad, medido dentro de la cámara de aquietamiento de la canaleta Parshall, y 3) el caudal de agua, calculado con la ecuación 1 y los valores de la tabla 3 , referentes a la canaleta propia de este proceso y utilizando el valor de $h$ correspondiente.

Figura 4. Valores para calcular el caudal según dimensiones de la canaleta

\begin{tabular}{||c|c|c||c|c||}
\hline \multicolumn{3}{|c||}{ W } & \multicolumn{2}{c||}{ K } \\
\cline { 1 - 1 } S.I. & \multirow{2}{*}{ m. } & $\mathbf{n}$ & $\begin{array}{l}\text { unidades } \\
\text { métricas }\end{array}$ & $\begin{array}{l}\text { unidades } \\
\text { inglesas }\end{array}$ \\
\hline \hline $\mathbf{3}^{\prime \prime}$ & 0.076 & 1.547 & 0.176 & 0.992 \\
\hline $\mathbf{6}^{\prime \prime}$ & 0.152 & 1.580 & 0.381 & 2.06 \\
\hline $\mathbf{9}^{\prime \prime}$ & 0.229 & 1.530 & 0.535 & 3.07 \\
\hline $\mathbf{1}^{\prime}$ & 0.305 & 1.522 & 0.690 & 4.00 \\
\hline $\mathbf{1}^{\prime} \mathbf{2}^{\prime}$ & 0.457 & 1.538 & 1.054 & 6.00 \\
\hline $\mathbf{2}^{\prime}$ & 0.610 & 1.550 & 1.426 & 8.00 \\
\hline $\mathbf{3}^{\prime}$ & 0.915 & 1.566 & 2.182 & 12.00 \\
\hline $\mathbf{4}^{\prime}$ & 1.220 & 1.578 & 2.935 & 16.00 \\
\hline $\mathbf{5}^{\prime}$ & 1.525 & 1.587 & 3.728 & 20.00 \\
\hline
\end{tabular}


$18 \circlearrowright$ Revista de investigación \#ashtag

O-

En la figura 5 se muestran las medidas de la in-

el uso de los valores de la figura 4 se obtienen los fraestructura relacionada con este proceso. Con valores registrados en la tabla 3.

Tabla 3. Valores del caudal mínimo y máximo en la Canaleta Parshall

\begin{tabular}{|l|l|}
\hline \multicolumn{2}{|c|}{ Datos canaleta Parshall } \\
\hline Ancho de garganta W & $2^{\prime}$ (pies), o $61 \mathrm{~cm}$ \\
\hline Altura máxima & $0,9 \mathrm{~m}$ \\
\hline Valor variable $\mathrm{K}$ & 1,426 \\
\hline Valor variable $\mathrm{n}$ & 1,55 \\
\hline
\end{tabular}

\begin{tabular}{|l|l|l|l|}
\hline \multicolumn{1}{|c|}{ Caudal Q } & \multicolumn{1}{c|}{$\mathbf{l} / \mathbf{s}$} & $\mathbf{m}^{3} \mathbf{s}$ & $\mathbf{m}^{3} / \mathbf{h}$ \\
\hline Máximo & 1210 & 1,2 & 4320 \\
\hline Mínimo & 117 & 0,117 & 421 \\
\hline
\end{tabular}

Fuente: elaboración propia

Figura 5. Plano general de la canaleta Parshall

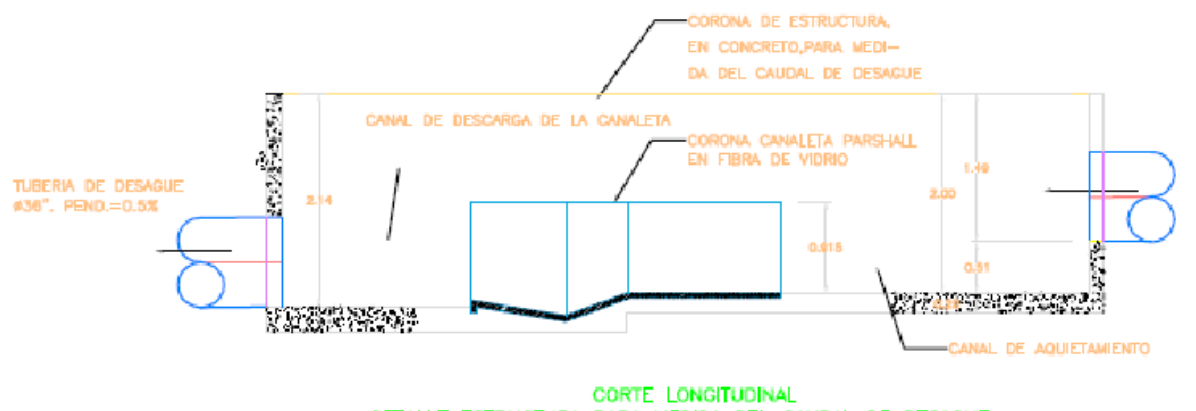

DETALLE ESTRUCTURA PARA MEOIDA DEL CAUDAL DE DESAGUE

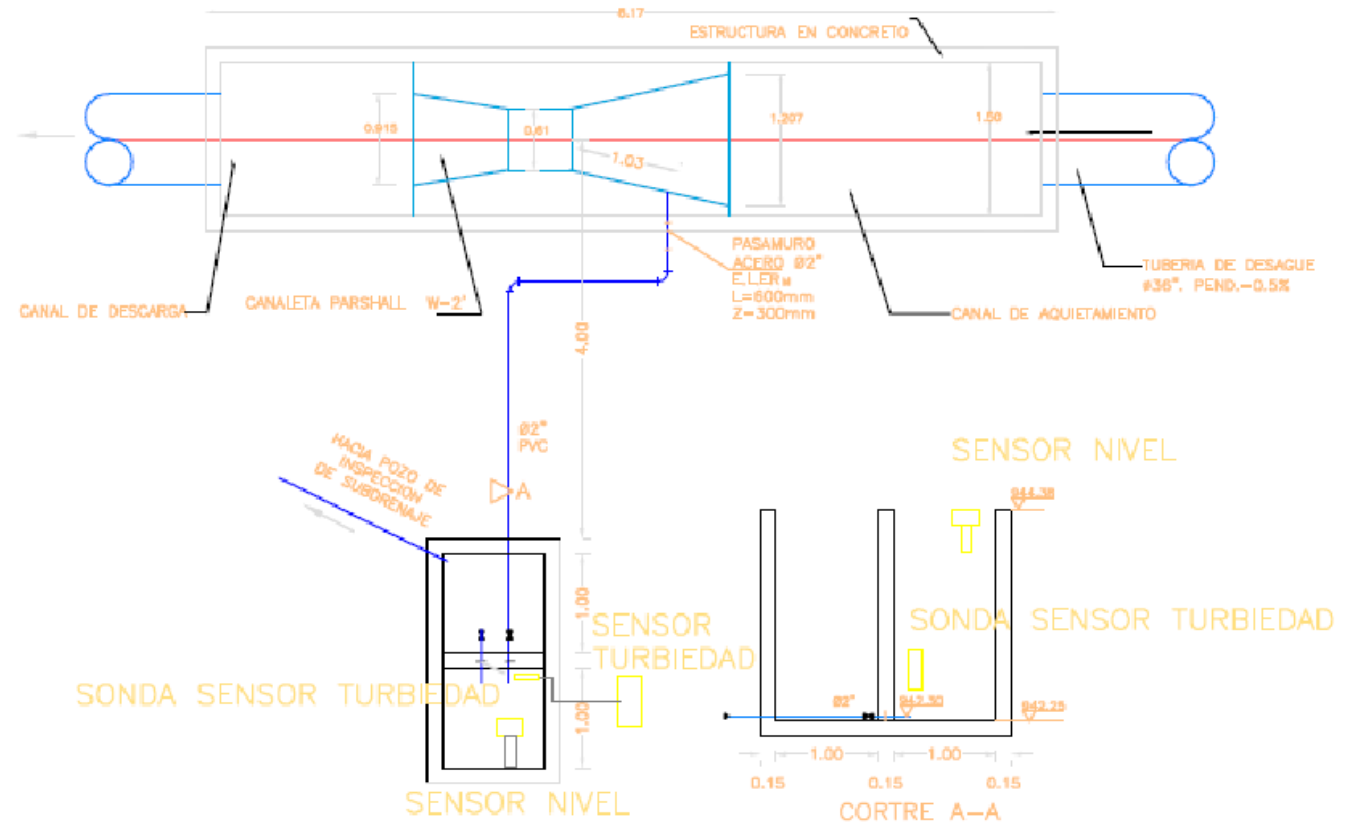

Fuente: elaboración propia 


\section{Sensor de nivel ultrasónico}

Este sensor fue seleccionado, según las existencias, en el almacén de repuestos de la ЕАAB. Su elección tuvo en cuenta las dimensiones de la canaleta Parshall (figura 5) y el hecho de que el

\section{Implementación del sensor}

Debido a la turbulencia y a la fuerza con la que ingresa el agua a la canaleta, el sensor debe instalarse en una cámara de aquietamiento próxima a la canaleta (figura 7) para que pueda capturar

\section{Sensor de turbiedad}

Este sensor fue seleccionado, según las existencias ,en el almacén de la EAAB. Su elección tuvo en cuenta que el controlador principal (PLC) tiene un módulo de comunicación Profibus y el nivel máximo de turbiedad permitida; es decir,

\section{Implementación del sensor}

Debido a la turbulencia y a la fuerza con la que ingresa el agua, el sensor debe instalarse en una cámara de aquietamiento, próxima a la canaleta de la figura 5, para que pueda hacer medidas controlador principal (PLC) dispone de un módulo de comunicación Profibus. Así, el sensor implementado es el Prosonic M FMU40.

medidas más adecuadas del nivel del agua. Este sensor hace parte de la red profibus implementada y su dirección es la 01. La figura 6 presenta un esquema de dicha red.

el valor mínimo de turbiedad en el agua para circular hacia la quebrada y el valor máximo de turbiedad en el agua que transita por la canaleta. Así, el sensor implementado es el Liquisys M CUM 223.

más adecuadas de la turbiedad del agua. Este sensor hace parte de la red PROFIBUS, detallada en la figura 6 , y su dirección es la 02.

Figura 6. Esquema de la red PROFIBUS

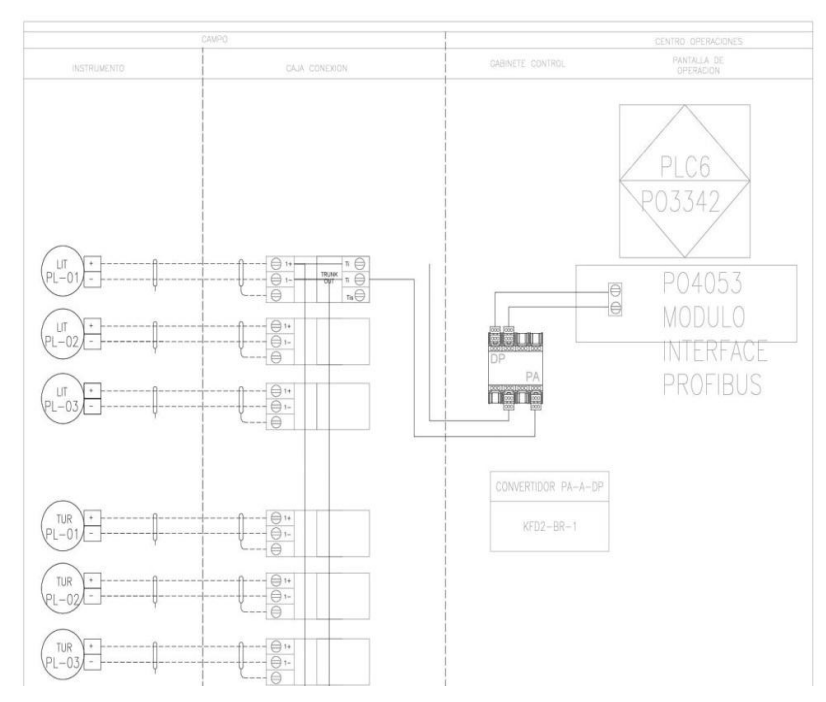




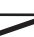

\section{Alarma 1: furbiedad mínima}

Como su nombre lo indica, esta alarma se genera cuando el nivel de turbiedad es mínimo -menor a 100 NTU-, es decir, es el adecuado para seguir su curso directo hacia la quebrada. Esta alarma se puede dar en dos ocasiones:

1. Cuando el agua ya está clarificada por el último lavado de los filtros.

2. Cuando hay exceso de agua y los procesos de potabilización no permiten el ingreso de más

\section{Intervención en la etapa 2}

En los tanques ecualizadores había dos sensores de nivel tipo flotador que generaban alarmas de nivel muy bajo. Por falta de mantenimiento, se encuentran en pésimas condiciones y no cumplen con la función para la cual fueron instalados. Con el ánimo de cumplir este requerimiento se implementaron dos sensores de nivel agua. Entonces, se excluye el agua sobrante y se permite el paso directo a la quebrada.

En estos casos, se genera una alarma en la interfaz hombre-máquina (HMI) del cuarto de lodos (figura 7) y una alarma en el Supervisorio General, con el fin de que un operario abra la válvula manual que da paso al agua hacia la quebrada. ultrasónicos, uno en cada tanque, para determinar el nivel máximo. También se utilizan los sensores de nivel para generar una alarma cuando el agua alcance el nivel mínimo y, así mismo, detener el funcionamiento de las bombas y proteger su integridad.

Figura 7. Ubicación del cuarto de lodos y HMI

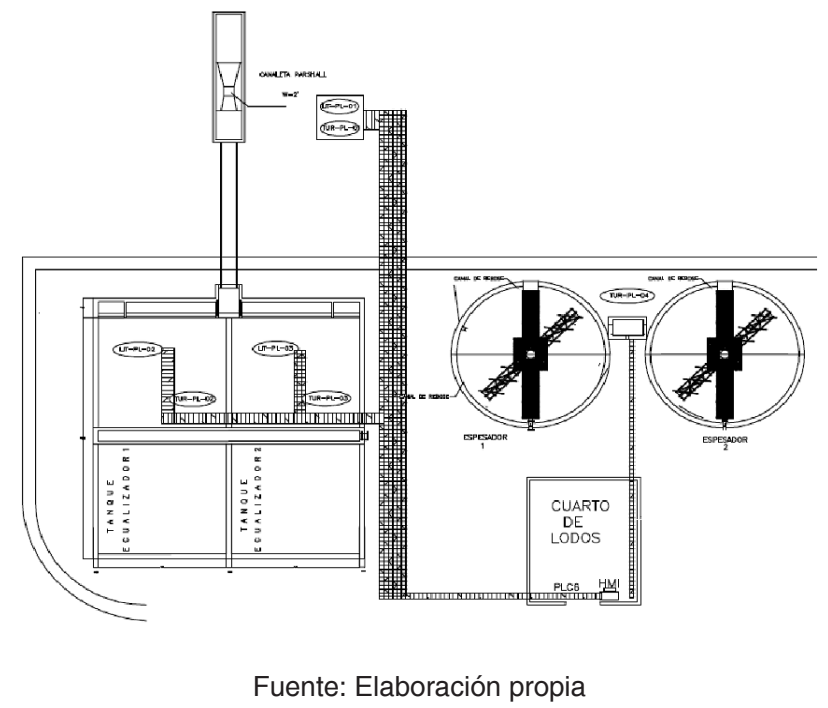

Además, este proceso tampoco cuenta con la instrumentación necesaria para determinar cuándo debe pasar el agua hacia la quebrada o cuándo debe pasar al siguiente proceso. Como se señaló anteriormente, esta decisión depende del criterio del operario, quien, en muchas ocasiones, 
debe tomar una muestra de agua con un dispositivo rudimentario de fabricación manual (figura 8) para llevarla al laboratorio de química de la planta y analizar la turbiedad.

\section{Sensor de nivel ultrasónico}

Este sensor, uno por cada tanque, fue seleccionado, según las existencias, en el almacén de la EAAB. Su elección tuvo en cuenta las dimensiones de los tanques de ecualizado (figura 5) y que el
Las variables de este proceso son: 1) el nivel del agua, medida desde la parte central de la superficie de cada tanque, y 2) el nivel de turbiedad, medida a 4,10 m de la superficie de cada tanque.

controlador principal (PLC) tiene un módulo de comunicación Profibus. Así, el sensor implementado es el Prosonic M FMU40.

Figura 8. Vasija para tomar la muestra de turbiedad

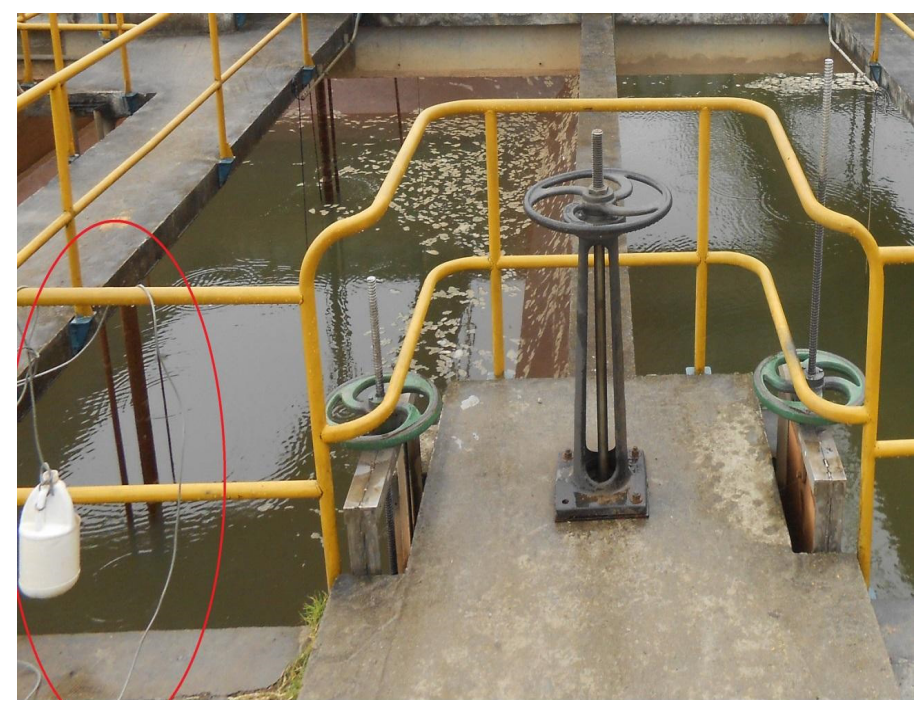

Fuente: elaboración propia

\section{Implementación del sensor}

Cada uno de estos sensores fue instalado en la superficie de su respectivo tanque, en el borde de la pasarela (figura 9). Estos sensores hacen

\section{Sensor de turbiedad}

Este sensor, uno por tanque, fue seleccionado, según las existencias, en el almacén de repuestos de la ЕАAB. Su elección tuvo en cuenta que el controlador principal (PLC) tiene un módulo de comunicación PROFIBUS y el nivel máximo de turbiedad parte de la red PROFIBUS y sus direcciones correspondientes son la 03 y 04 (figura 6).

permitida -el valor mínimo de turbiedad en el agua hacia la quebrada y el valor máximo que puede tomar la turbiedad del agua que circula hacia el proceso en los tanques espesadores-. Así, el sensor instalado es el Liquisys M CUM 223. 


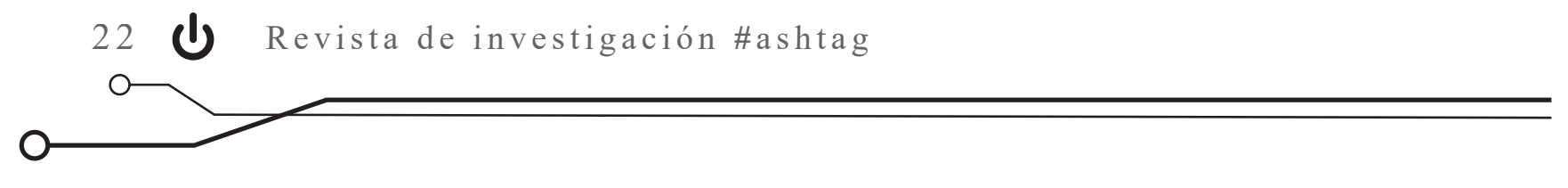

\section{Implementación del sensor}

La sonda de cada uno de los sensores de turbiedad se instala a 4,10 m, suspendidos desde el borde superior central de los tanques y asegurados a la pasarela. Los controladores de cada sensor se ajustan en el borde de la pasarela, tal como se detalla en la figura 9. Estos sensores hacen parte de la red PROFIBUs y sus direcciones son la 05 y 06 (figura 6).

Figura 9. Instalación de los sensores de turbiedad

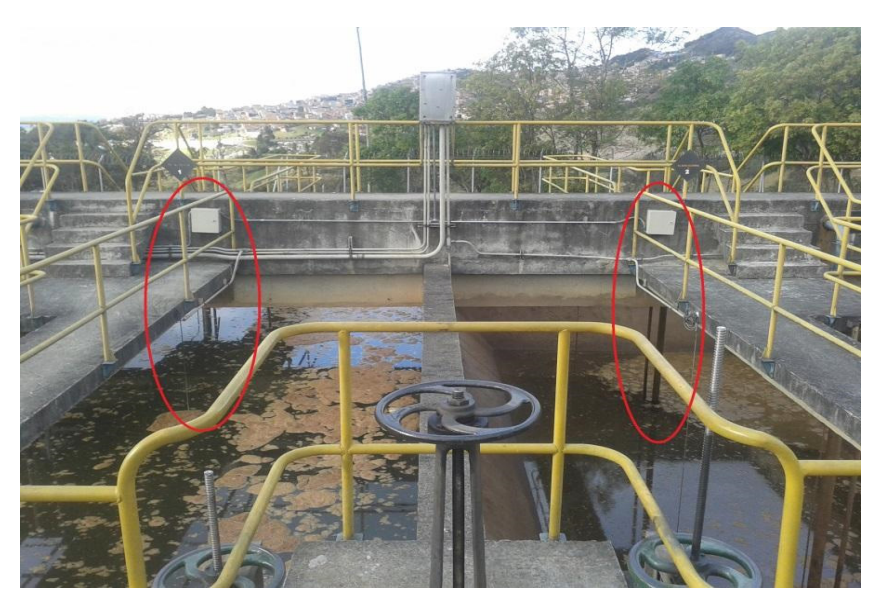

Fuente: elaboración propia

\section{Alarma 1: turbiedad mínima}

Como su nombre lo indica, esta alarma se genera cuando el nivel de turbiedad es mínimo -menor a 50 NTU-, es decir, el adecuado para seguir su curso directo hacia la quebrada. Esta alarma se puede dar en tres ocasiones:

1. Cuando el agua ya está clarificada por el último lavado de los filtros.

2. Cuando hay exceso de agua y los procesos de potabilización no permiten el ingreso de más agua. Entonces, el restante se excluye, de tal

\section{Alarma 2: nivel mínimo}

Esta alarma se genera cuando el agua dentro de los dos tanques alcanza el nivel mínimo. Esta indica que los motores deben ser apagados de manera que el agua sobrante pasa directo a la quebrada.

3. Cuando el proceso en los tanques ecualizadores se ha llevado a cabo y se ha enviado el lodo hacia los tanques espesadores. Entonces, el agua clarificada debe ser enviada a la quebrada.

Para los tres casos anteriores, se genera una alarma en el HMI del cuarto de lodos (figura 7) y una alarma en el SCADA, con el fin de que un operario abra la válvula manual que da paso al agua hacia la quebrada.

manera automática para evitar daños por funcionamiento en el vacío. Esta alarma genera un indicador en el HMI del cuarto de lodos y en el SCADA. 


\section{Alarma 3: nivel máximo}

Esta alarma se genera cuando el agua dentro de los dos tanques alcanza el nivel máximo. Esta indica a los operarios que se debe cerrar la compuerta principal para evitar el rebose de agua sin clarificar. Esta alarma genera un indicador en el HMI del cuarto de lodos y en el SCADA.

\section{Intervención en la etapa 3}

En los tanques espesadores hay cuatro válvulas manuales, dos por cada tanque, y a diferente altura. Estas válvulas se encuentran conectadas a la sonda de un sensor de turbiedad o al vaso de muestreo indicado en la figura 10.

Figura 10. Instrumentación en los tanques espesadores

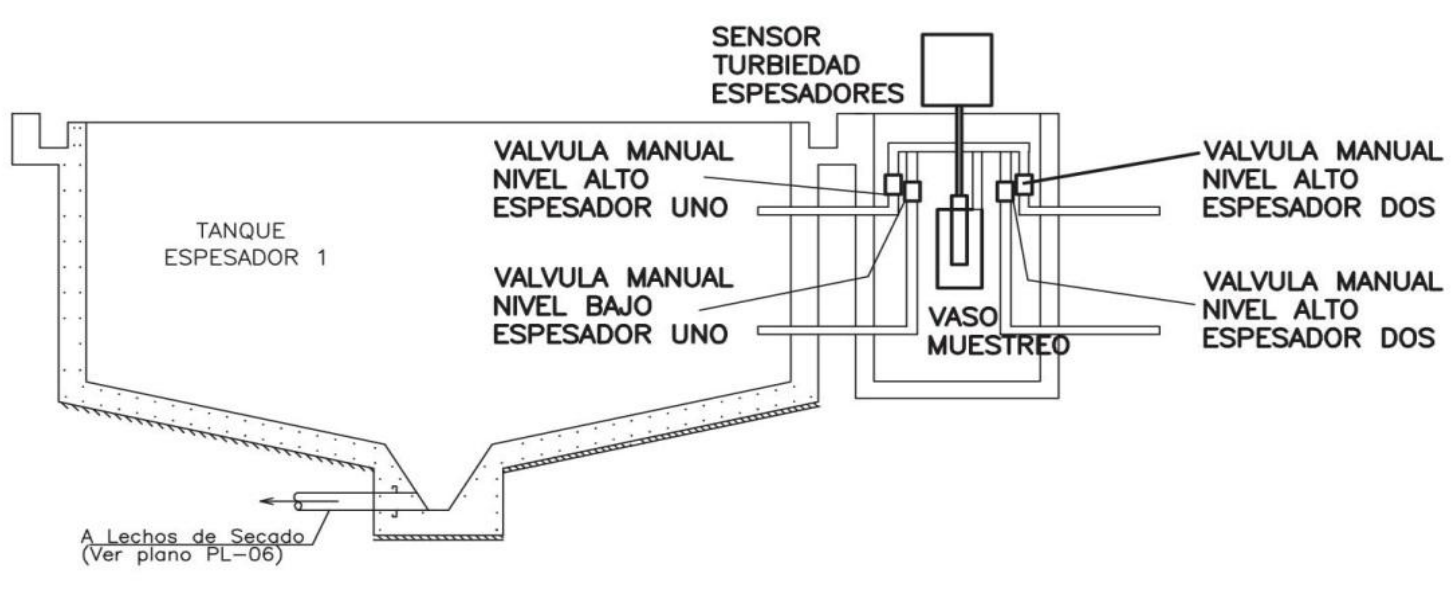

Fuente: elaboración propia

Este sistema se utiliza para tomar la turbiedad en los diferentes puntos de manera manual, al abrir una válvula a la vez. Cabe recordar que dichas válvulas, y su respectiva tubería, estaban obstruidas por lodo seco, pues no habían recibido mantenimiento.

De acuerdo a la turbiedad obtenida en el fondo, se da el paso hacia la fase dos. Como el sistema de medición se encontraba obstruido, el operador, por simple inspección, toma la decisión correspondiente, sin tener la certeza de que la calidad del agua que pasa a la quebrada, por efecto del rebose, sea la adecuada ni que el lodo que pasa hacia la segunda fase tenga la turbiedad necesaria.

La variable de este proceso es el nivel de turbiedad, medido a 4,10 $\mathrm{m}$ de la superficie de cada tanque para validar la concentración de lodo en el fondo. También se mide a $30 \mathrm{~cm}$ de la superficie para monitorear la calidad del agua que es evacuada hacia la quebrada. 


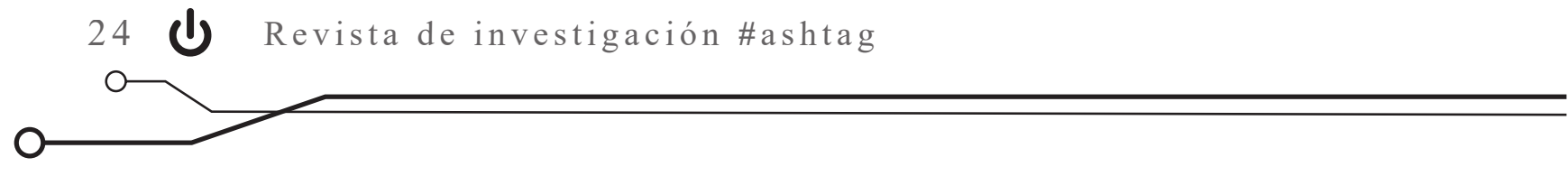

\section{Sensor de turbiedad}

Este sensor, uno para los dos tanques, ya estaba instalado. Tras su respectivo mantenimiento, se conectó a la red PROFIBUS del sistema.

El sensor instalado es el Liquisys M CUM 223. Para implementarlo se realizó el necesario mantenimiento de las tuberías y las válvulas, dos por

\section{Alarma 1: nivel turbiedad para la quebrada}

Como su nombre lo indica, esta alarma se genera cuando el nivel de turbiedad en la parte superior es mínimo -menor a 50 NTU-, es decir, el adecuado para seguir su curso directo hacia la quebrada. Esta alarma se genera con el fin de continuar con el bombeo de lodo desde los cada tanque. Dos de ellas se ubican el nivel superior de cada tanque y las otras dos en el nivel inferior. Estas se conectan directamente con la sonda de medición del turbidímetro (figura 10). Este sensor hace parte de la red PROFIBUs y su dirección es la 06 (figura 6).

\section{Alarma 2: nivel de turbiedad alto para la quebrada}

Esta alarma se genera cuando el nivel de turbiedad en la parte superior de los espesadores es muy alto -mayor a 50 NTU- para que el agua tanques ecualizadores y así pueda continuar la acción de rebose. Cuando esta alarma se genera, de manera automática se enciendan los motores del bombeo de los ecualizadores y se muestra su acción en el HMI del cuarto de lodos y en el SCADA del supervisorio general de la planta.

\section{Alarma 3: nivel de turbiedad alto para fase 2}

Esta alarma se genera cuando el nivel de turbiedad en la parte inferior de los espesadores es muy alto -mayor a 500 NTU-, es decir, el valor indicado para que el lodo circule hacia la fase dos. Cuando esta alarma se genera, se indica en

\section{Supervisorio (SCADA)}

El sistema SCADA, desarrollado e implementado, se realizó con el software Indusoft ${ }^{\circledR}$ Web Studio, debido a que la planta cuenta con la licencia de este producto. Además, el SCADA de toda la planta de tratamiento de agua potable El Dorado también esta soportado por esta misma plataforma. circule hacia la quebrada. Cuando esta Alarma se genera, de manera automática se detiene el bombeo desde los ecualizadores. el HMI y en el SCADA para que el operador realice la apertura de las válvulas que llevan el lodo hacia la segunda fase. Además, detiene el bombeo desde los ecualizadores.

La comunicación se efectuó mediante Ethernet TCP/IP (Geronimo, 2005) y el entorno, en general, se diseñó para permitirle al usuario supervisar todos los sensores, el caudal de la canaleta Parshall, el estado de operación de las bombas sumergibles en los tanques ecualizadores, su 
nivel de agua, la turbiedad en estos y en los tanques espesadores, así como las alarmas anteriormente descritas. En la figura 11 se puede observar un mímico general de todo el proceso de clarificación.

Figura 11. Sistema SCADA del proceso de clarificación de agua en la planta de lodos

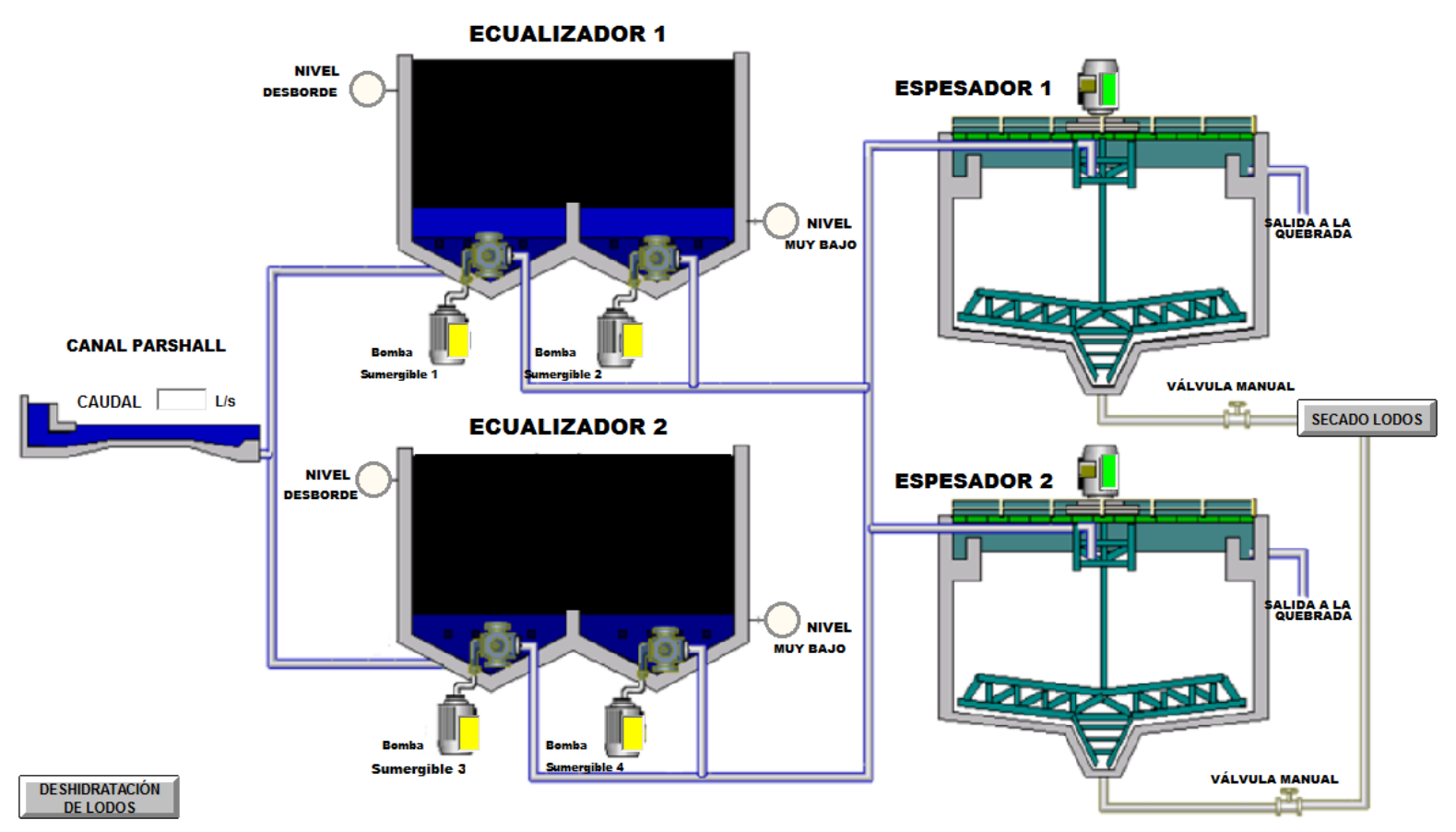

Fuente: elaboración propia

\section{Conclusiones}

Pese a encontrar grandes limitaciones de índole económico y administrativo en la planta El Dorado de la $\mathrm{EAAB}$, que no permitieron realizar un sistema totalmente automatizado, el sistema implementado cumple con los requisitos de funcionamiento del proceso de lodos, que se pretendía en este proyecto. Dicho sistema fue implementado, en parte, reutilizando equipos que habían salido de funcionamiento en otras plantas de la EAAB, y así se solucionaron los problemas encontrados en las tres etapas del proceso de clarificación.
El sistema implementado monitorea constantemente la turbiedad del agua hacía la quebrada, lo que mejora notablemente su calidad y evita la afectación de las poblaciones y cultivos vecinos que usan este líquido, porque el 100 \% del agua clarificada que se dispone hacía la quebrada está por debajo del nivel requerido por el EAAB, es decir, menos de 100 NTU.

Gracias a la implementación del proyecto, los ingenieros encargados de la supervisión ahora cuentan con un sistema SCADA con el que pueden monitorear constantemente las distintas etapas 


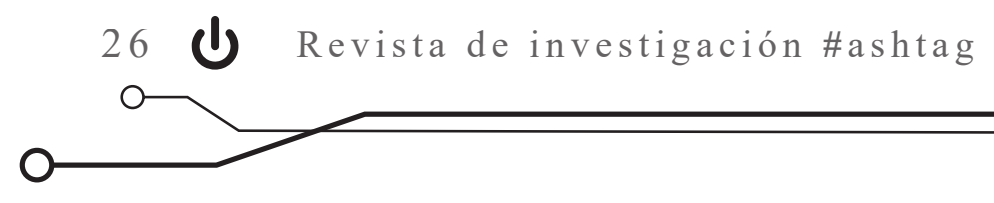

del proceso y, a su vez, ejercer control sobre la intervención oportuna que deben realizar los operadores cuando el proceso así lo requiera, abriendo o cerrando compuertas.

Una vez puesto en marcha el proyecto, el tiempo de permanencia de los operarios que supervisaban presencialmente el proceso se redujo. Previamente, todo el proceso tardaba aproximadamente 7 horas y ahora tarda aproximadamente 5 horas, de acuerdo a la turbiedad y volumen del agua. El tiempo del operario supervisor del proceso se redujo, lo que da pie para ayudar a otras actividades de tratamiento de agua potable. Antes de la puesta en marcha del proyecto, el proceso de clarificación se realizaba dos veces al día; ahora, con los sensores de turbiedad implementados, se pueden realizar hasta tres procesos por día.

Se observó una reducción en el mantenimiento correctivo en las bombas sumergibles, pues antes se realizaba una vez al mes. Con el sistema implementado no ha sido necesario realizar ningún mantenimiento correctivo desde hace tres meses.

Con el sistema y el algoritmo implementado en las etapas canaleta Parshall y en los tanques ecualizadores se logró mejorar el homogenizado de la mezcla, tanto de lodo como de agua. Por lo general, y de acuerdo a él, volumen y turbiedad del agua las 2/3 partes son de agua clarificada y $1 / 3$ de lodo, para continuar a la siguiente etapa.

Al disponer de controladores PO3342 de Altus (Altus, 2011b) -de los cuales no hay mucha información en la web o en libros, y el fabricante no ofrece soporte si no hay un contrato de mantenimiento establecido- se utilizó la metodología de ensayo y error en diversas pruebas realizadas, mientras nos apoyábamos en la lectura constante de los manuales (Altus, 2001, y Altus, 2011a). Se lograron integrar todos dispositivos necesarios para el proyecto, además de configurarlos para la correcta ejecución de tareas en los requisitos establecidos.

La interfaz hombre-máquina permitió supervisar, integrar, identificar y sugerir el estado de dispositivos totalmente operativos por seres humanos en el sistema de supervisión, $\mathrm{y}$, en efecto, para realizar un mejor proceso de automatización; por ejemplo, con las compuertas y válvulas de acceso y salida de líquidos o lodos (Sistemas Digitales de Control 2002 SL, 2014).

La implementación de una red industrial, como lo es PROFIBUS, permitió, en gran medida, el desarrollo del proyecto, pues gracias a este protocolo se logró disminuir el costo del presupuesto y se ahorró en cableado y en costo de adquisición de nuevos módulos para el PLC Altus. Así mismo, facilitó la implementación y configuración de la instrumentación requerida para cada una de las etapas, lo que demuestra que es una herramienta de alto nivel para procesos industriales a gran escala.

La inclusión de la comunicación vía Ethernet (Geronimo, 2005) facilitó ampliamente conseguir la integración del proceso de clarificación con el supervisorio general de la planta El Dorado. Para ello se empleó el software Indusoft $\mathrm{t}^{\circledR}$.

El grado de automatización conseguido durante el proceso de clarificación de agua, si bien no fue total, sirvió para mejorar la calidad del agua que fluye hacia los torrentes naturales y para mejorar la consistencia del lodo restante que ingresa a la siguiente fase, es decir, la deshidratación.

El sistema de alarmas integrado al proyecto, además de cumplir con cuidar la integridad de 
los elementos y del proceso, permitiría a futuro, de acuerdo a un presupuesto asignado, actualizar o reestructurar este proceso, y adquirir equipos necesarios para la automatización total que puedan ser integrados al sistema sin mayores inconvenientes (Sistemas Digitales de Control 2002 SL, 2014).

\section{Referencias}

Acueducto. (2015). Agua y Alcantarillado de Bogotá. Recuperado de http://www.acueducto. com.co/wpsv61/wps/portal

Altus. (2001). Manual de Utilização da Série Ponto [recurso en línea]. Recuperado de http:/ /www. altusarg.com.ar/manuales/Mu209000.pdf

Altus. (2011a). Características e Configuração da Série Ponto [recurso en línea]. Recuperado de http://dualtechautomacao.com.br/Produtos/CT109000.pdf

Altus. (2011b). Manual de Utilização PO3042/PO3142 PO3242/PO3342 UCPs Série PONTO, MU209104 [recurso en línea]. Recuperado de https://www.altus.com.br/suporte/ download/baixararquivo/AQEH/2

Ambientum. (2002). Deshidratación de fangos. Revista Ambientum, Recuperado de http:// www.ambientum.com/revista/2002_40/DSHDRTCN1.asp

Endress+Hauser. (2006). Turbidity/Suspended Solids Measurement Liquisys M CUM 223 / 253. Technical Information, TI 200C/07/en, No. 51500283 [recurso en línea]. Recuperado de http:// www.merteh.lv/eh/pdf/TI200CEN.PDF

Endress+Hauser. (2008). Prosonic M FMU40/41/42/43/44 - Medición de nivel ultrasónica. Información técnica, TI365F/23/es [recurso en línea]. Recuperado de https://portal.endress.com/ wa001/dla/5000000/1439/000/01/TI365Fes_v04.07.pdf

Geronimo, G. (2005). Ethernet y protocolos TCP/IPv4 [recurso em línea]. Recuperado de https:// silo.tips/download/ethernet-y-protocolos-tcp-ipv4

Lux, M. (2010). Medidores de flujo en canales abiertos [trabajo de grado]. Universidad de San Carlos de Guatemala, Guatemala. Recuperado de http://biblioteca.usac.edu.gt/tesis/08/08_3165_C.pdf

Reyes, W. (2004). Evaluación de la eficiencia de la planta de tratamiento para la potabilización de agua del municipio de Cerrito (Santander) [trabajo de especialización]. Universidad Industrial de Santander, Bucaramanga, Colombia. Recuperado de http://tangara.uis.edu. co/biblioweb/tesis/2004/114456.pdf 


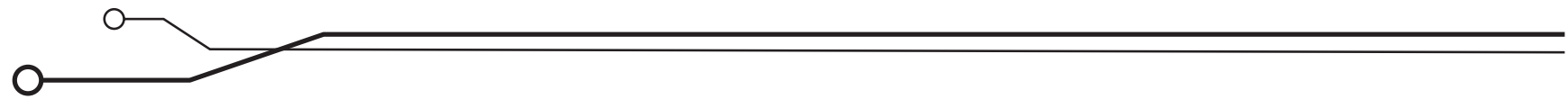

Santis, M. y Villa, E. (2009). Estudio del protocolo Fielbus y aplicación práctica con el controlador SMAR DF51 para el manejo de instrumentos industriales [trabajo de especialización]. Universidad Pontificia Bolivariana, Bucaramanga, Colombia. Recuperado de https://repository.upb. edu.co/bitstream/handle/20.500.11912/523/digital_17957.pdf

Sistemas Digitales de Control 2002 SL. (2014). Desarrollo de software sCADAS [recurso en línea]. Recuperado de http://www.sistemas2002.com/Area_Industrial/Scada/

Suárez, J., Jácome, A. y Ures, P. (2015). Tecnologías de espesamiento (FT-FNG-001). Serie: tratamiento de fangos. España: Universidad de la Coruña; INDITEx. Recuperado de https:// www.wateractionplan.com/documents/177327/558161/Tecnologías+de+espesamiento. pdf/5937d248-06a9-c654-cd44-4583aca5acdb

Tecnología Digital del Bajío. (2012). ¿Qué es el MODBUS? MODBUS parte III [entrada de blog]. Recuperado de https:/ / es.scribd.com/ document/239880944/2-Modbus-Parte-III 